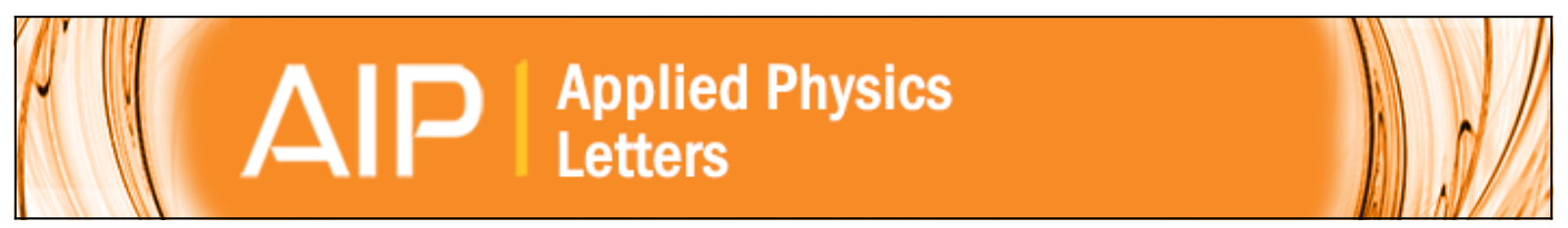

\title{
Ultra-thin filaments revealed by the dielectric response across the metal-insulator transition in VO2
}

J.-G. Ramírez, Rainer Schmidt, A. Sharoni, M. E. Gómez, Ivan K. Schuller, and Edgar J. Patiño

Citation: Applied Physics Letters 102, 063110 (2013); doi: 10.1063/1.4792052

View online: http://dx.doi.org/10.1063/1.4792052

View Table of Contents: http://scitation.aip.org/content/aip/journal/apl/102/6?ver=pdfcov

Published by the AIP Publishing

\section{Articles you may be interested in}

Analysis of diverging effective mass extracted from thermoelectric power across the metal-insulator transition in VO2

J. Appl. Phys. 118, 035105 (2015); 10.1063/1.4926860

Comprehensive study of the metal-insulator transition in pulsed laser deposited epitaxial VO2 thin films J. Appl. Phys. 113, 043707 (2013); 10.1063/1.4788804

Evolution of local work function in epitaxial VO2 thin films spanning the metal-insulator transition Appl. Phys. Lett. 101, 191605 (2012); 10.1063/1.4766292

Heteroepitaxial VO2 thin films on GaN: Structure and metal-insulator transition characteristics J. Appl. Phys. 112, 074114 (2012); 10.1063/1.4758185

Geometric confinement effects on the metal-insulator transition temperature and stress relaxation in VO2 thin films grown on silicon

J. Appl. Phys. 109, 063512 (2011); 10.1063/1.3556756

Frustrated by

old technology?

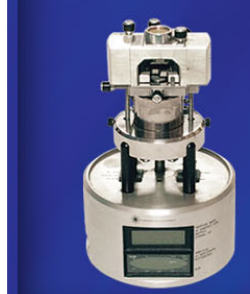

Is your AFM dead

and can't be repaired?

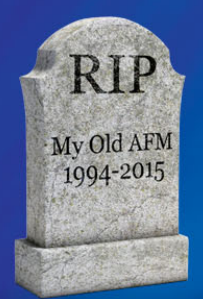

Sick of bad customer support?

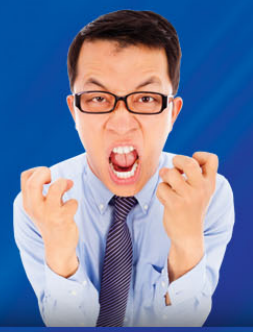

It is time to upgrade your AFM Minimum $\$ 20,000$ trade-in discount for purchases before August 31st

Asylum Research is today's technology leader in AFM 


\title{
Ultra-thin filaments revealed by the dielectric response across the metal-insulator transition in $\mathrm{VO}_{2}$
}

\author{
J.-G. Ramírez, ${ }^{1,2}$ Rainer Schmidt, ${ }^{3}$ A. Sharoni, ${ }^{4}$ M. E. Gómez, ${ }^{5}$ Ivan K. Schuller, ${ }^{2}$ \\ and Edgar J. Patiño ${ }^{1}$ \\ ${ }^{1}$ Departamento de Física, Grupo de Física de la Materia Condensada, Universidad de los Andes, Bogotá, \\ Colombia \\ ${ }^{2}$ Department of Physics and Center for Advanced Nanoscience, University of California-San Diego, \\ La Jolla, California 92093, USA \\ ${ }^{3}$ Departamento Física Aplicada III, GFMC, Universidad Complutense de Madrid, \\ Facultad de Ciencias Físicas, 28040 Madrid, Spain \\ ${ }^{4}$ Department of Physics, Bar-Ilan University, Ramat-Gan 52900, Israel \\ ${ }^{5}$ Thin Film Group, Universidad del Valle A.A.25360, Cali, Colombia
}

(Received 3 October 2012; accepted 30 January 2013; published online 13 February 2013)

\begin{abstract}
Temperature dependent dielectric spectroscopy measurements on vanadium dioxide thin films allow us to distinguish between the resistive, capacitive, and inductive contributions to the impedance across the metal-insulator transition (MIT). We developed a single, universal, equivalent circuit model to describe the dielectric behavior above and below the MIT. Our model takes account of phase-coexistence of metallic and insulating regions. We find evidence for the existence at low temperature of ultra-thin threads as described by a resistor-inductor element. A conventional resistorcapacitor element connected in parallel accounts for the insulating phase and the dielectric relaxation. (C) 2013 American Institute of Physics. [http://dx.doi.org/10.1063/1.4792052]
\end{abstract}

Materials that undergo a metal-insulator phase transition (MIT) often exhibit unconventional electronic properties. Since in many cases these transitions are first order, inhomogeneity and phase coexistence play a crucial role. ${ }^{1-3}$ Since these may be the basis for novel electronic devices, a fundamental understanding of the transition mechanism and the concomitant changes in the dielectric properties are crucial to optimize device performance.

Vanadium dioxide $\left(\mathrm{VO}_{2}\right)$ exhibits a prototypical MIT, with the following key features: (1) A sharp structural-phase transition from a low-temperature, insulating, monoclinic phase to a high-temperature, metallic, rutile phase occurs. ${ }^{1}$ (2) Phase-coexistence of metallic and insulating domains at the nano-scale near the MIT leads to percolation and avalanches. ${ }^{4,5}$ (3) The MIT can be induced with light, pressure, and an electric field, which changes the phase-equilibrium of the coexisting phases. ${ }^{1}$

All these properties may be utilized in potential applications of $\mathrm{VO}_{2}$ in the electronic industry, for example, in the next-generation resistive nonvolatile random access memories (RAM). ${ }^{6}$

The phase-coexistence in $\mathrm{VO}_{2}$ across the MIT is particularly intriguing, because the metallic phase starts to form locally far below the macroscopic MIT in the form of persistent metallic domains within the insulating matrix. ${ }^{7}$ It was argued from current-voltage measurements that a thermal filament or thread forms at large enough bias voltage (see for example Ref. 8 and references therein). The thread diameter depends on the applied electric field, which manipulates the equilibrium between conducting threads and surrounding insulating areas. Several decades ago the formation of $\mathrm{VO}_{2}$ conducting threads was simulated using an equivalent circuit in which the threads were modeled by a series resistor-inductor $\left(\mathrm{R}_{\mathrm{X}} \mathrm{L}_{\mathrm{X}}\right)$ element connected in parallel with a standard resistor, where the latter accounts for the surrounding insulating matrix. ${ }^{9}$ This approach, however, was not followed up possibly because it ignores all capacitive contributions to the dielectric properties. Therefore, a coherent and satisfactory description of the complex dielectric data across the $\mathrm{VO}_{2}$ transition is still not available. Here, we show that the series $R_{X} L_{X}$ element properly represents the conducting threads if it is connected in parallel to a standard RC element. Such standard RC element consists of a resistor and capacitor connected in parallel, and is well known to be of fundamental importance for the description of standard dielectric relaxations. ${ }^{10}$ The equivalent circuit used here can account for the in-plane dielectric response of $\mathrm{VO}_{2}$ thin films. Below the MIT, the model reveals the existence of a highly inductive component. This inductance is a direct proof of the existence of ultra-thin conducting threads. Above the MIT, it describes large percolating conducting areas, which is reflected by a large inductance. These results have implications for the theoretical understanding of the MITs in general. In addition, our model provides a framework for quantitative analysis of the $\mathrm{VO}_{2}$ MIT and its dielectric response, and how it may affect the performance of electronic components based on these materials.

We deposited $\mathrm{VO}_{2}$ thin films by reactive $\mathrm{RF}$ magnetron sputtering from a $99.8 \%$ vanadium target (1.5 in. diameter) on r-cut (1012) sapphire substrates. The deposition was carried out in a high-vacuum chamber with a base pressure of $5 \times 10^{-8}$ Torr using a mixture of ultra-high purity (UHP) argon and oxygen sputtering gases. The single-phase $\mathrm{VO}_{2}$ films were characterized by X-ray diffraction using $\mathrm{CuK} \alpha$ radiation and energy-dispersive $\mathrm{X}$-ray spectroscopy. The measurements indicate that the $\mathrm{VO}_{2}$ is textured along the $(00 l)$ plane and is highly crystalline. A nearly five orders of magnitude change in the dc resistance close to $330 \mathrm{~K}$ signals the MIT, consistent with previous work. ${ }^{4}$ 


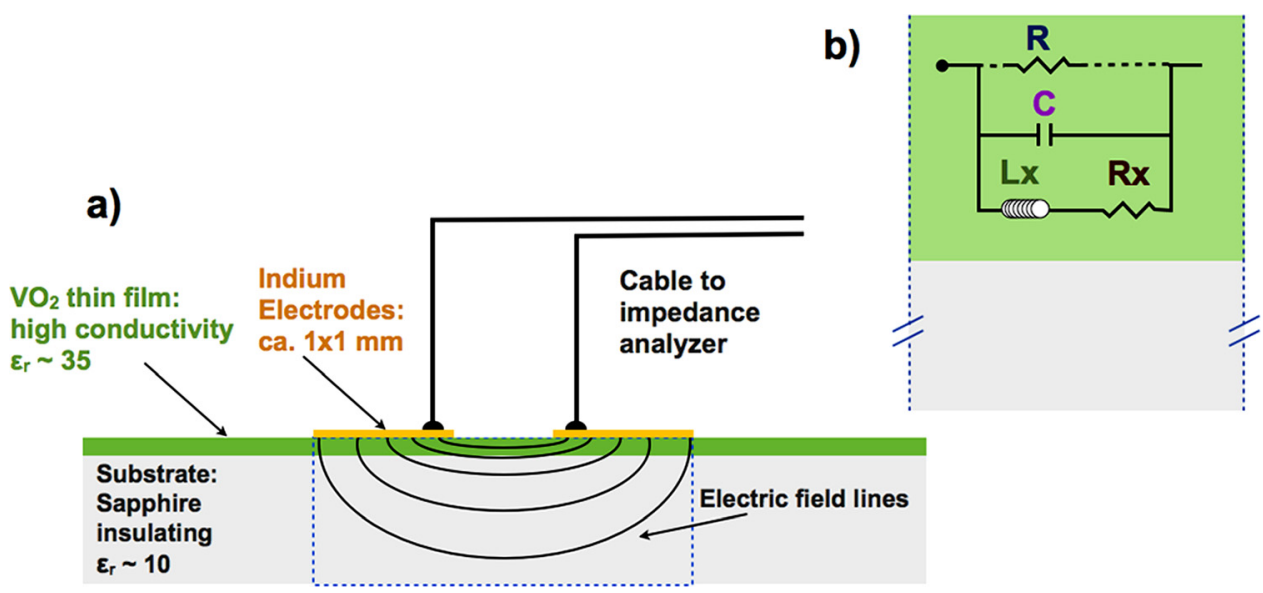

FIG. 1. (a) Measurement setup for inplane impedance spectroscopy of a $90 \mathrm{~nm}$-thick $\mathrm{VO}_{2}$ thin film at a frequency range $100 \mathrm{~Hz}-10 \mathrm{MHz}$ between 290 and $345 \mathrm{~K}$. (b) Equivalent circuit of the device. $\mathrm{R}-\mathrm{C}$ models the insulating contributions to the total impedance, whereas the $R_{x} L_{x}$ branch models the inductive response from the sample.

In-plane impedance spectroscopy (IS) was performed using an HP $4194 \mathrm{~A}$ Impedance analyzer in the $100 \mathrm{~Hz}$ to $10 \mathrm{MHz}$ frequency range on a custom-built temperaturecontrolled sample stage. A $0.5 \mathrm{~V}$ voltage amplitude produced a low signal-to-noise ratio. Copper wires $(0.1 \mathrm{~mm}$ diameter and $10 \mathrm{~cm}$ length) were attached to the sample using $(99.99 \%$ pure) indium contacts. Prior to connecting to the sample, open-circuit and short-circuit tests were performed to minimize parasitic contributions from the measurement setup. Conventionally, IS measurements are performed in out-ofplane parallel plate capacitor configuration, where the electric field lines are mostly perpendicular to the film surface. ${ }^{11}$ However, in $\mathrm{VO}_{2}$ films this set-up is inadequate, because it results in low film resistance below the resolution limit above the MIT. The alternative in-plane arrangement of top-top electrodes leads to higher and measurable resistance values due to the larger macroscopic distance between the electrodes (see Fig. 1(a)). The long current path across the sample is shown here to be also an ideal way to probe the formation of ultrathin conducting threads.

IS is a well-established tool for the characterization of a large range of solid-state phenomena such as MITs, e.g., in $\mathrm{ZnO}$ nano-powders, ${ }^{12}$ for probing resistive switching in $\mathrm{NiO}$ and $\mathrm{TiO}_{2}$ thin films, ${ }^{13-15}$ measure magneto-impedance in $\mathrm{MgO}$ tunnel barriers ${ }^{16}$ and $\mathrm{BiMnO}_{3}$ thin films, ${ }^{17}$ and determine surface passivation in silicon. ${ }^{18}$ In each case, IS gives relevant information about the resistive, capacitive, and inductive behaviors of separated intrinsic and extrinsic dielectric relaxation processes in the sample.

Fig. 2 shows IS spectra collected below the MIT $\left(T_{\mathrm{C}} \approx 333 \mathrm{~K}\right)$ upon heating, where the data are plotted as the negative imaginary vs. the real part of the impedance $-Z^{\prime \prime}$ vs. $Z^{\prime}$. In such complex impedance plane plots, one or more semicircles occur in case of a perfect parallel plate capacitor measurement; one semicircle each for one series dielectric relaxation such as extrinsic Maxwell-Wagner interface and intrinsic film contributions. ${ }^{10}$ Careful examination of Fig. 2 reveals that at intermediate/high frequencies, the semicircles are slightly extended on the $-Z^{\prime \prime}$ axes, i.e., somewhat "elongated," which is atypical for parallel plate capacitor measurements and cannot be modeled solely using standard RC elements.

Therefore, we used the alternative equivalent circuit depicted in Fig. 1(b), which produces excellent fits at all frequencies as indicated by the solid lines. Physically, the R-C branch accounts for the insulating phase within the film, where the use of a standard RC element points towards a standard dielectric behavior. The unusually "elongated" behavior can be fitted by adding a series $\mathrm{R}_{\mathrm{X}} \mathrm{L}_{\mathrm{X}}$ branch, in parallel to the conventional RC element (Fig. 1(b)). As mentioned above, the use of the $\mathrm{R}_{\mathrm{X}} \mathrm{L}_{\mathrm{X}}$ branch has been suggested before to account for the coexistence of conventional insulating areas and conducting domains in the films. ${ }^{7,9}$ The parallel arrangement of the conventional $\mathrm{RC}$ and additional $\mathrm{R}_{\mathrm{X}} \mathrm{L}_{\mathrm{X}}$ branch may suggest a predominantly parallel arrangement of such two phases.

The IS collected above the MIT is presented in Fig. 3 on $-Z^{\prime \prime}$ vs. $Z^{\prime}$ axes. The imaginary impedance $Z^{\prime \prime}$ is now positive, because the spectra are dominated by the inductor $\mathrm{L}_{\mathrm{X}}$. The model remains the same except that now the resistor $\mathrm{R}$ is too large (compared to the other impedances), and is therefore not reflected in the spectra. Percolation of large metallic domains across the film is the simple explanation for the lack of sensitivity to the large insulating resistor $\mathrm{R}$, where the conducting areas are no longer filamentary.

The validity of our universal model is further assessed in Fig. 4, where the real part of the conductivity is plotted versus frequency, $f .^{19}$ The MIT appears as a conductivity jump from $\approx 8 \times 10^{-6}$ at $330 \mathrm{~K}$ to $\approx 1.8 \times 10^{-2}$ at $337 \mathrm{~K}$. In this notation, the curves taken above and below the MIT resemble each other, justifying the use of the universal circuit model. In both cases, the curves show two distinctively different regimes separated by the dotted line in Fig. 4: (A) At low/intermediate frequency, the $\sigma^{\prime}$ vs. $f$ curves are

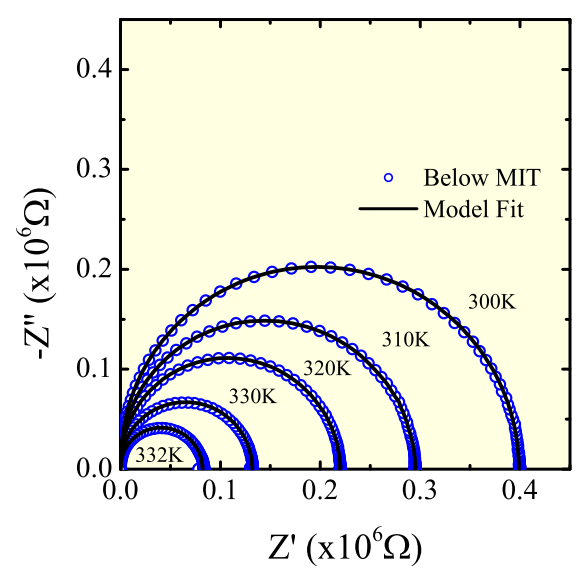

FIG. 2. IS data collected below the MIT, plotted as the negative imaginary vs. real part of the impedance $\left(-Z^{\prime \prime}\right.$ vs. $\left.Z^{\prime}\right)$. Open symbols indicate the data; solid (black) lines represent the fit of the data to the model shown in Fig. 1(b). 


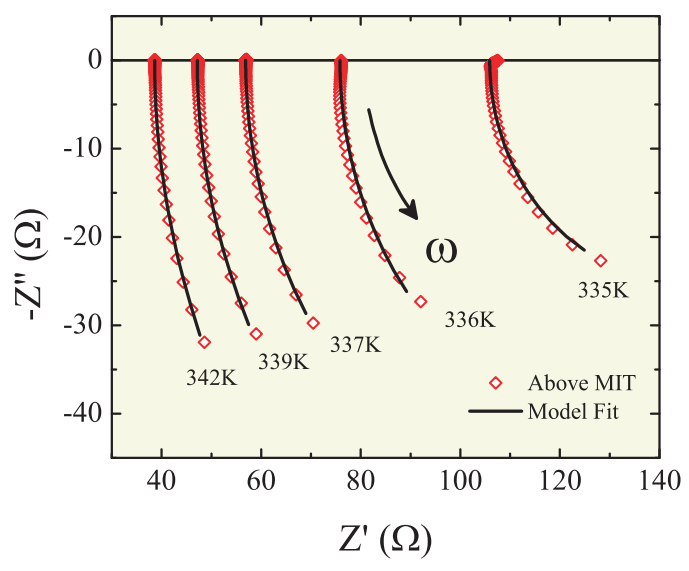

FIG. 3. IS data collected above the MIT, plotted as the negative imaginary vs. real part of the impedance $\left(-Z^{\prime \prime}\right.$ vs. $\left.Z^{\prime}\right)$. Open symbols indicate the data; solid (black) lines represent the fit of the data to the model shown in Fig. 1(b).

approximately $f$ independent, indicative of a standard dielectric relaxation as represented by an RC element. (B) At intermediate/high frequency, $\sigma^{\prime}$ vs. $f$ curves show a decrease, which is unusual and inconsistent with standard dielectric theory in terms of the brick-work layer model, applicable to classical parallel-plate capacitor measurement used for dielectric materials. ${ }^{16}$ Such behavior is typical of inductive contributions, leading to decreasing conductivity at high $f$. At low/intermediate frequency, the electrical current may flow in parallel through the insulating (RC) and the conducting $\left(\mathrm{R}_{\mathrm{X}} \mathrm{L}_{\mathrm{X}}\right)$ domains. At higher $f$, the conducting domains exhibit increasing impedance due to inductive effects $\left(\mathrm{L}_{X}\right)$ and the current passes mainly across the insulating film areas, leading to the observed drop in $\sigma^{\prime}$ at $\operatorname{high} f$.

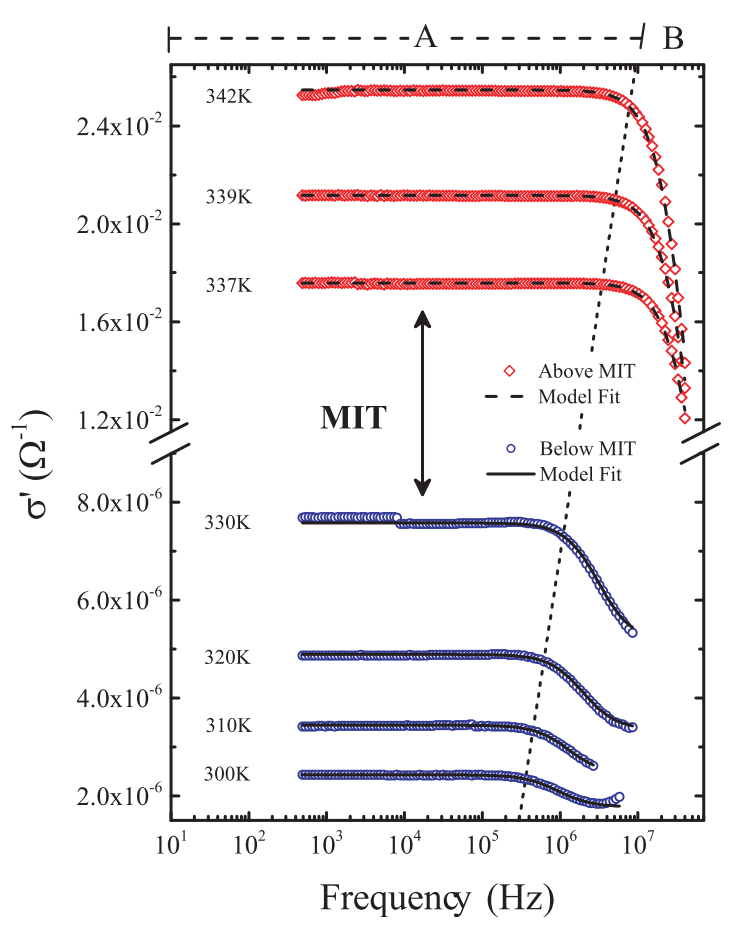

FIG. 4. IS data collected below and above the MIT, plotted as real part of the conductivity $\sigma^{\prime}$ vs. $f$. Open symbols indicate the data, solid and dashed lines represent the fit of the data to the model shown in Fig. 1(b), below and above the MIT, respectively. The data fall into two frequency regimes (regimes $\mathrm{A}$ and $\mathrm{B}$ ) as indicated by the dotted line. The MIT appears as a jump from $\approx 8 \times 10^{-6} \Omega^{-1}$ to $\approx 1.6 \times 10^{-2} \Omega^{-1}$.
Figures 2-4 demonstrate unambiguously that the dielectric data presented as impedance $-Z^{\prime \prime}$ vs. $Z^{\prime}$ and conductivity $\sigma^{\prime \prime}$ vs. $f$ can be well modeled by our RC- $\mathrm{R}_{\mathrm{X}} \mathrm{L}_{\mathrm{X}}$ circuit leading to a good fit at all frequencies across the MIT. In order to gain further confidence in our model, we attempted to forcefit the data to a single RC model at low and intermediate $f$, which produced significantly higher data scatter in the R vs. $\mathrm{T}$ curve than for our advanced model. This is a further strong indication for the need of our universal model, including two resistors, capacitor, and inductor.

All fitted parameters extracted from our model are shown in Fig. 5, where each point in the curves represents a fit at a specific temperature with the fitting-convergence criteria $\left(\chi^{2}\right)$ kept below $10^{-4}$. The vertical dotted line (red online) at $334 \mathrm{~K}$ separates the two different regions below and above the MIT. The equivalent sample resistance $R_{E Q}$ for all temperatures was calculated from the fitted resistor values according to $1 / R_{\mathrm{EQ}}=1 / \mathrm{R}+1 / \mathrm{R}_{\mathrm{X}}$ and compared to the dc resistance as shown in Fig. 5(a). $R_{\mathrm{EQ}}$ below and above the MIT agrees quantitatively with the independently measured $\mathrm{dc}$ resistance, which lends further confidence to the correctness of our model. Above the MIT, we used $\mathrm{R}_{\mathrm{EQ}}=\mathrm{R}_{\mathrm{X}}$.

Below the MIT, $\mathrm{R}$ and $\mathrm{R}_{\mathrm{X}}$ decrease monotonically as the temperature increases as shown in Figs. 5(b) and 5(c). Once the MIT is reached, $R_{X}$ undergoes a sharp drop by more than four orders of magnitude as a manifestation of the breakdown of the insulating matrix.

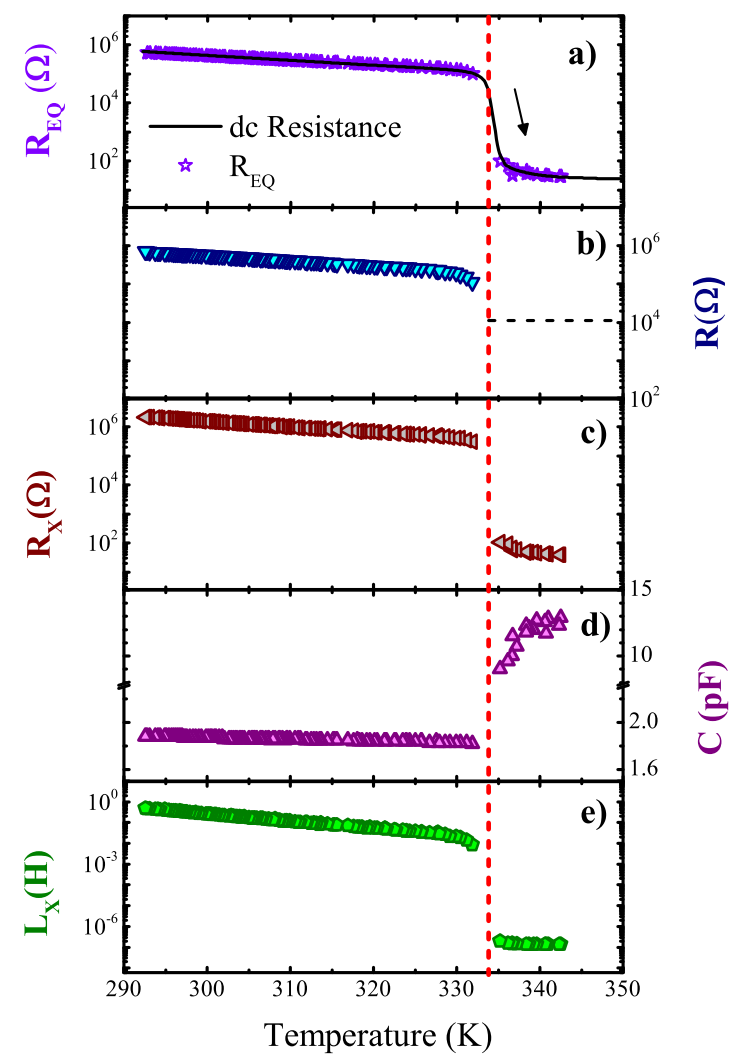

FIG. 5. Temperature dependences of the equivalent circuit elements. The vertical dashed line (red online) indicates the MIT. (a) dc resistance measurements of the $\mathrm{VO}_{2}$ thin film (solid line). The equivalent resistance $\mathrm{R}_{\mathrm{EQ}}$ (open stars), obtained from the $R$ and $R_{X}$ values according to: $1 / R_{E Q}=1 / R+1 / R_{X}$. (b) Insulating resistance $\mathrm{R}$ for $T<T_{\mathrm{C}}$. (c) Resistance $\mathrm{R}_{\mathrm{X}}$ for conducting areas undergoing a change of almost four orders of magnitude. (d) Capacitance $\mathrm{C}$. (e) Inductance $\mathrm{L}_{\mathrm{X}}$, showing a large drop across the MIT. 
It is interesting to note that $\mathrm{R}$ and $\mathrm{R}_{\mathrm{X}}$ are of the same order of magnitude below the transition. One may assume low resistance of the "conducting" phase $\mathrm{R}_{\mathrm{X}}$, which is not what we observe. $R_{X}$ appears in fact quite similar to the insulating areas $\mathrm{R}$, which may confirm previous claims that the conducting phase may form ultra-thin threads. ${ }^{20}$ It was further suggested that these filaments grow with temperature, in agreement with the standard phase-coexistence present in a 1st order phase transition. ${ }^{11,21,22}$ From these arguments and our findings, it seems plausible that below the MIT electrical current may flow both within the conducting filaments and across the insulating film areas in a parallel configuration, which may highlight the competition between insulating and conducting domains and explain the similar resistance in $\mathrm{R}$ and $\mathrm{R}_{\mathrm{X}}$. Above the MIT, the model provides a lower limit for $\mathrm{R}\left(10^{4} \Omega\right)$, indicated by the dashed line in Fig. 5(b).

Fig. 5(d) depicts the device capacitance C, extracted from the model, which slightly decreases as temperature increases and then shows a sharp upturn at the MIT. Interpretation of the capacitive results is difficult, since the field lines of the applied ac electric field are not parallel (see Fig. 1) and $\mathrm{C}$ may contain contributions from the film and substrate. Furthermore, the changes in capacitance may not necessarily reflect intrinsic changes in the film dielectric permittivity, but could be caused by the development of filaments, which changes the effective current cross section between the two electrodes.

Fig. 5(e) depicts the temperature dependence of the inductance $\mathrm{L}_{\mathrm{X}}$. Below the MIT, a large $\approx 0.3 \mathrm{H}$ inductance is detected. This may be consistent with the presence of metallic domains in the $\mathrm{VO}_{2}$ film well below the MIT as suggested by earlier first order reversal curve (FORC) measurements. ${ }^{7}$ The slow decrease of $L_{X}$ below the MIT suggests that the conducting domains grow in size with increasing temperature. The large inductance drop by six orders of magnitude at the MIT may indicate the disappearance of the filaments above the MIT and percolation of large conducting domains as expected in the metallic phase.

In conclusion, in-plane IS measurements were performed in high quality $\mathrm{VO}_{2}$ thin films to investigate the temperature dependence of the frequency dependent dielectric response. A universal impedance model describes quantitatively the complex dielectric data above and below the MIT. The model includes a conventional RC circuit to account for insulating areas and an inductive branch $\mathrm{R}_{\mathrm{X}} \mathrm{L}_{\mathrm{X}}$ in parallel to describe filamentary conductive domains, which become dominant at higher frequencies. As a consistency check, the resistive part extracted from the model is in good quantitative agreement with independently measured dc resistance measurements. The equivalent capacitance increases and the inductance decreases substantially with increasing temperature across the MIT. These results confirm the existence of ultra-thin, highly conductive domains well below the MIT, which grow in size with increasing temperature and coalesce above the MIT.
In a broader sense, this work shows that traditional modeling of impedance spectroscopy ${ }^{9}$ needs modifications when applied to novel, modern materials, which in many cases are intrinsically inhomogeneous.

This work was supported by AFOSR Grant No. FA955012-1-0381, COLCIENCIAS, CENM and "El Patrimonio Autónomo Fondo Nacional de Financiamiento para la Ciencia, la Tecnología y la Innovación Francisco José de Caldas" Contract RC-No. 275-2011, and ISF Grant No. 727/11. R.S. wishes to acknowledge a Ramón y Cajal Fellowship from the Ministerio de Ciencia e Innovación (MICINN) in Spain. E.J.P. wishes to acknowledge "Programa Nacional de Ciencias Básicas" COLCIENCIAS (No. 120452128168). During the publication process of this manuscript, out-of-plane impedance measurements were published on $\mathrm{VO}_{2}$ films grown on $\mathrm{TiO}_{2}$ substrates. These results also imply the coexistence of metallic and insulating phases. ${ }^{22}$

${ }^{1}$ M. Imada, A. Fujimori, and Y. Tokura, Rev. Mod. Phys. 70, 1039 (1998).

${ }^{2}$ V. Y. Zerov, Y. V. Kulikov, V. N. Leonov, V. G. Malyarov, I. A. Khrebtov, and I. I. Shaganov, J. Opt. Technol. 66, 387 (1999).

${ }^{3}$ S. M. Babulanam, T. S. Eriksson, G. A. Niklasson, and C. G. Granqvist, Sol. Energy Mater. 16, 347 (1987).

${ }^{4}$ A. Sharoni, J. G. Ramírez, and I. K. Schuller, Phys. Rev. Lett. 101, 026404 (2008).

${ }^{5}$ M. M. Qazilbash, M. Brehm, B. G. Chae, P. C. Ho, G. O. Andreev, B. J. Kim, S. J. Yun, A. V. Balatsky, M. B. Maple, F. Keilmann, H.-T. Kim, and D. N. Basov, Science 318, 1750 (2007).

${ }^{6}$ M.-J. Lee, Y. Park, D.-S. Suh, E.-H. Lee, S. Seo, D.-C. Kim, R. Jung, B.-S. Kang, S.-E. Ahn, C. B. Lee, D. H. Seo, Y.-K. Cha, I.-K. Yoo, J.-S. Kim, and B. H. Park, Adv. Mater. 19, 3919 (2007).

${ }^{7}$ J. G. Ramírez, A. Sharoni, Y. Dubi, M. E. Gomez, and I. K. Schuller, Phys. Rev. B 79, 235110 (2009).

${ }^{8}$ A. Zimmers, L. Aigouy, M. Mortier, A. Sharoni, S. Wang, K. G. West, J. G. Ramirez, and I. K. Schuller, Phys. Rev. Lett. 110, 056601 (2013).

${ }^{9}$ C. N. Berglund, IEEE Trans. Electron Devices 16, 432 (1969).

${ }^{10} \mathrm{E}$. Barsoukov and J. R. Macdonald, Impedance Spectroscopy: Theory, Experiment, and Applications (John Wiley \& Sons, Inc., Hoboken, New Jersey, 2005).

${ }^{11}$ R. Schmidt, W. Eerenstein, T. Winiecki, F. D. Morrison, and P. A. Midgley, Phys. Rev. B 75, 245111 (2007).

${ }^{12}$ M. Nadeem, A. Farooq, and T. J. Shin, Appl. Phys. Lett. 96, 212104 (2010).

${ }^{13}$ Y. H. You, B. S. So, J. H. Hwang, W. Cho, S. S. Lee, T. M. Chung, C. G. Kim, and K. S. An, Appl. Phys. Lett. 89, 222105 (2006).

${ }^{14}$ M. H. Lee, K. M. Kim, G. H. Kim, J. Y. Seok, S. J. Song, J. H. Yoon, and C. S. Hwang, Appl. Phys. Lett. 96, 152909 (2010).

${ }^{15}$ Z. Yang, C. Ko, V. Balakrishnan, G. Gopalakrishnan, and S. Ramanathan, Phys. Rev. B 82, 205101 (2010).

${ }^{16}$ S. Ingvarsson, M. Arikan, M. Carter, W. Shen, and G. Xiao, Appl. Phys. Lett. 96, 232506 (2010).

${ }^{17}$ R. Schmidt, W. Eerenstein, and P. A. Midgley, Phys. Rev. B 79, 214107 (2009).

${ }^{18}$ S. Kumar, P. K. Singh, and S. R. Dhariwal, Appl. Phys. Lett. 96, 162109 (2010).

${ }^{19}$ The real part of the conductivity is defined as $\sigma^{\prime}=Z^{\prime} /|\mathrm{Z}|$.

${ }^{20}$ C. N. Berglund and R. H. Walden, IEEE Trans. Electron Devices 17, 137 (1970).

${ }^{21}$ J. Duchene, M. Terraillon, P. Pailly, and G. Adam, Appl. Phys. Lett. 19, 115 (1971).

${ }^{22}$ X. Zhong, P. LeClair, S. Sarker, and A. Gupta, Phys. Rev. B 86, 094114 (2012). 